\title{
Twelve cases of drug-induced blepharospasm improved within 2 months of psychotropic cessation
}

This article was published in the following Dove Press journal:

Drug, Healthcare and Patient Safety

2 June 2011

Number of times this article has been viewed

Yuko Emoto'

Hirofumi Emoto ${ }^{2}$

Eriko Oishi'

Syunichi Hikita'

Masato Wakakura'

'Division of Neuro-Ophthalmology, Inouye Eye Hospital, Tokyo;

${ }^{2}$ Department of Ophthalmology and

Visual Science, Tokyo Medical and

Dental University, Graduate School of

Medicine, Tokyo, Japan
Correspondence: Masato Wakakura Inouye Eye Hospital, 4-3 Kanda-

Surugadai, Chiyoda-ku, Tokyo I0I-0062, Japan

Tel +8I3 3295 09। I

$\mathrm{Fax}+81332950917$

Email wakakura-m@inouye-eye.or.jp
Background: To determine whether psychotropic cessation in patients with drug-induced blepharospasm improves motor symptoms.

Methods: In patients with drug-induced blepharospasm, we withdrew part or all of their psychotropic medication and assessed motor symptoms using the Jankovic rating scale $(0=$ none, $1=$ noticeable, $2=$ mild, $3=$ moderate, $4=$ severe) at first presentation and after cessation.

Results: Twelve patients (eleven women and one man, mean age 60.4 years) were enrolled. Psychotropics were administered before the onset of blepharospasm in all patients. The mean duration of treatment with psychotropic medication was 47.3 (range 3-120) months. Jankovic rating scale at initial presentation was 3 in eleven patients and 2 in one patient. After cessation, blepharospasm started to improve in all cases within 2 months (average 3.9 weeks). While the effect of psychotropic cessation was variable, the symptoms eventually improved to more than 2 on the rating scale. Three of the twelve patients underwent a single botulinum neurotoxin injection and were withdrawn from therapy after cessation.

Conclusion: Psychotropic drugs can cause blepharospasm in some cases. Clinicians should consider reducing psychotropic medication as far as possible in patients with blepharospasm taking these agents.

Keywords: drug-induced, tardive, blepharospasm, antipsychotic, dose reduction, benzodiazepine

\section{Introduction}

Blepharospasm is a form of focal dystonia characterized by three major symptoms, ie, motor symptoms (difficulty keeping the eyes open and excessive blinking), sensory symptoms (irritation of the eyes and photophobia), and psychiatric symptoms (depression and anxiety). ${ }^{1,2}$ The disorder is rarely self-limiting and generally is a lifelong disability. ${ }^{3}$

Blepharospasm can be classified into three groups, ie, essential, secondary, and druginduced. Drug-induced blepharospasm is most commonly associated with neuroleptics, as well as dopaminergic agents, antihistamines, calcium channel blockers, and noradrenaline and serotonin reuptake inhibitors. ${ }^{4}$ Additionally, we have shown that benzodiazepines and thienodiazepines can often induce blepharospasm. ${ }^{5}$ We report here twelve cases of drug-induced blepharospasm that improved after psychotropic cessation.

\section{Methods}

Patients were recruited from the Division of Neuro-Ophthalmology at Inouye Eye Hospital, Japan, between 2004 and 2009. Patients were included in the study if they 
had been on psychotropic medication that started after the onset of blepharospasm. Exclusion criteria were: refusal of consent to enrollment after being informed about the risks and benefits of psychotropic cessation; decision not to cease the drug or reduce the dose following communication with psychiatrists; lack of follow-up for at least 6 months after cessation; presence of motor symptoms from other movement disorders, including Parkinsonism, dyskinesia, and akathisia; and presence of major psychiatric diseases, such as bipolar disorder or schizophrenia that required psychotropic medication to be used.

Patients who met the selection criteria were enrolled consecutively. After obtaining informed consent from the patients, and permission from the psychiatrists or internists who prescribed the patient's medication, we gradually reduced the dose of the psychotropics. During dose reduction, patients were closely monitored by their neuro-ophthalmologists and psychiatrists. The study was approved by the institutional ethics committee.

The motor symptoms of blepharospasm were assessed using the Jankovic rating scale, whereby $0=$ none, $1=$ noticeable, $2=$ mild, $3=$ moderate, and $4=$ severe, at the initial presentation and during dose reduction. ${ }^{6}$ We performed a final symptom assessment using the same scale at least 6 months after we completed psychotrophic cessation.

We also obtained information for each patient, including age, gender, family history, past medical history, pharmacological history, the time interval between onset of symptoms and when the patients were diagnosed as having blepharospasm, the time interval between when the psychotropic medication was initiated and when we started to reduce the dose, and the psychiatric diagnoses for which the psychotropics were prescribed.

\section{Results}

Of 138 patients with drug-induced blepharospasm, twelve consecutive patients who met the criteria were enrolled (eleven females and one male, Table 1). There were no patients who had a significant family history, previous neurological disorder, or any other movement disorders except for blepharospasm. No patients received any other therapies that may have influenced central nervous system function, including hormone replacement therapy. Mean age of the 12 patients was 60.4 years, with a range of 36-76 years (Table 2). The mean time interval from the onset of blepharospasm to when the diagnosis was made was 10.8 (range 1-42) months. The Jankovic rating scale at the initial presentation was 3 in eleven patients and 2 in one patient. The final Jankovic rating after psychotrophic cessation was 1 in four patients and 0 in eight patients. The difference in rating on the scale between the initial and final assessment was 3 in seven patients and 2 in five patients. The most commonly prescribed psychotropics withdrawn were etizolam (five patients) followed by brotizolam, alprazolam, and zolpidem (one patient each). The mean duration of treatment with psychotropic medication was 47.3 (range 3-120) months. The psychiatric diagnoses were insomnia (eleven patients), depression (seven patients), and anxiety (six patients). On average, symptoms started to improve 3.9 weeks after cessation (range 1-8 weeks). While the effect of psychotropic cessation was variable, the symptoms eventually improved to more than 2 on the rating scale, with $67 \%(8 / 12)$ of the patients completely recovering.

Cases 10, 11, and 12 received botulinum neurotoxin injection therapy (Table 1, Figure 1). Cases 10 and 11 received the injection after dose reduction was started, while case 12 had an injection prior to dose reduction. These three cases improved after receiving a single injection, and therefore decided to stop therapy once dose reduction was completed.

\section{Case presentation}

A 57-year-old woman (case 7 in Table 1) presented with a 2-year history of difficulty keeping her eyes open, excessive blinking, eye irritation, and photophobia. For the previous 3 years and 9 months, she had been continuously treated with etizolam $4 \mathrm{mg} /$ day for insomnia and anxiety. After 16-18 months of medication, she felt marked fatigue of her eyes. Voluntary closure of the eyes and sleep relieved this symptom. She tried to avoid sunlight and wind because of eye irritation. Her friends could not understand why she could not open her eyes and she began to avoid company. These symptoms made her more nervous and her insomnia got worse.

She consulted an ophthalmologist about her eye problem and was diagnosed as having "dry eye". The eye irritation did not improve even though she received treatment for it. Another ophthalmologist's review revealed no local pathology and her symptoms were thought to be of psychosomatic origin. Blinking became more troublesome approximately 6 months before presentation to our unit. Her psychiatrist noticed her symptoms at routine follow-up and referred her to our eye hospital.

On examination, she was noted to have excessive blinking and bilateral involuntary spasms of the orbicularis oculi muscles leading to partial eye closure lasting a few seconds. Other disorders, such as hemifacial spasm, myokymia, 


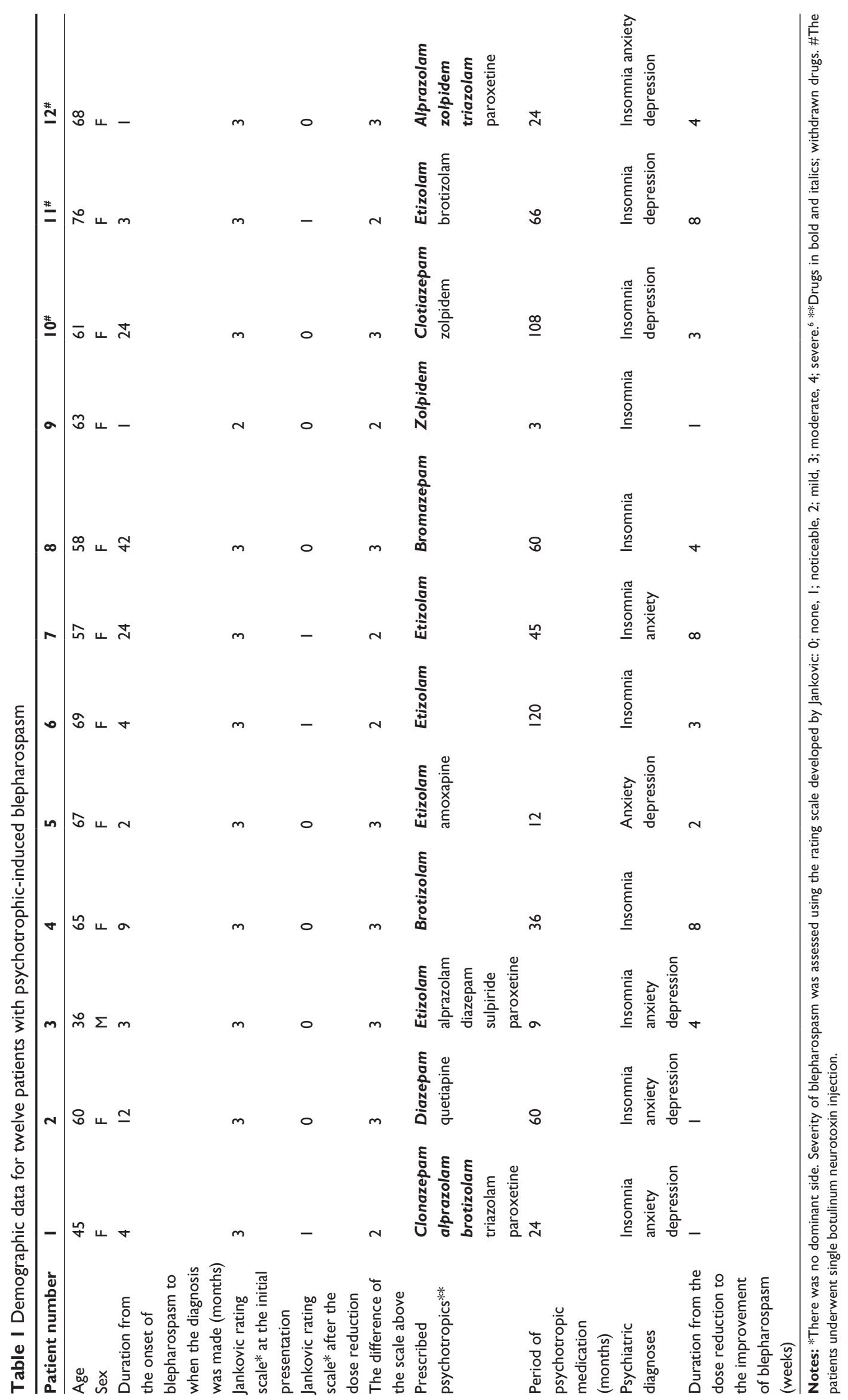


Table 2 Overview of the twelve patients

\begin{tabular}{|c|c|}
\hline Mean age & $60.4(36-76)$ \\
\hline Sex & II $\mathrm{F}$ and I $\mathrm{M}$ \\
\hline Mean duration from the onset of blepharospasm to when the diagnosis was made (months) & $10.8(I-42)$ \\
\hline Jankovic rating scale* at the initial presentation & $3: 11 \mathrm{pts}, 2: 1 \mathrm{pt}$ \\
\hline Jankovic rating scale* after the dose reduction & $\mathrm{I}: 4 \mathrm{pts}, 0: 8 \mathrm{pts}$ \\
\hline Difference of the scale above & 3:7 pts, $2: 5$ pts \\
\hline The most prescribed psychotropics & Etizolam brotizolam alprazolam zolpidem \\
\hline Mean period of psychotropic medication (months) & $47.3(3-120)$ \\
\hline Mean duration from the dose reduction to the improvement of blepharospasm (weeks) & $3.9(I-8)$ \\
\hline
\end{tabular}

Notes: *There was no dominant side. Severity of blepharospasm was assessed using the rating scale developed by Jankovic: 0; none, I; noticeable, 2; mild, 3; moderate, 4; severe. ${ }^{6}$ Abbreviations: $F$, female; $M$, male.

apraxia of eyelid opening, and myasthenia gravis, were carefully excluded by ancillary testing. There was no significant family history, previous neurological disorder, or any movement disorders (including Parkinsonism, dyskinesia, and akathisia) except for blepharospasm.

A diagnosis of blepharospasm caused by psychotropic medication was made. The Jankovic rating scale at initial presentation was 3 , ie, moderate in severity. We started to reduce to half dose in collaboration with her psychiatrist. During dose reduction, the patient was closely monitored by neuro-ophthalmologists and psychiatrists, and we evaluated her motor symptoms of blepharospasm using the Jankovic rating scale. Eight weeks later, she noticed she could open her eyes more easily than before. She experienced a temporary worsening of her insomnia, and the extent of her excessive blinking fluctuated depending on the day. Other treatments were not necessary because the symptom gradually improved after psychotropic reduction. Etizolam was gradually withdrawn 2 months after the start of dose reduction. One month after cessation, she was almost free from blepharospasm and the Jankovic scale rating was improved to 1 , ie, noticeable in severity.

\section{Discussion}

There were no patients affected by major psychosis in the current study, and all patients enrolled had only mild psychotic problems, such as anxiety, insomnia, or depression. Patients were on various benzodiazepines (alprazolam, bromazepam, clonazepam, diazepam, ethyl loflazepate, or triazolam) and thienodiazepines (brotizolam, clotiazepam, or etizolam), which are widely prescribed in Japan, primarily for insomnia and anxiety. The most commonly prescribed psychotropic agent was etizolam, a thienodiazepine derivative and a popular anxiolytic, with a high affinity for benzodiazepine receptors. We have previously reported that benzodiazepine and thienodiazepine can cause drug-induced blepharospasm, with early cessation of the drugs resulting in the relief of the motor symptoms of blepharospasm. ${ }^{5}$

In all cases in the current study, blepharospasm started to improve within 2 months (mean 3.9, range 1-8 weeks)

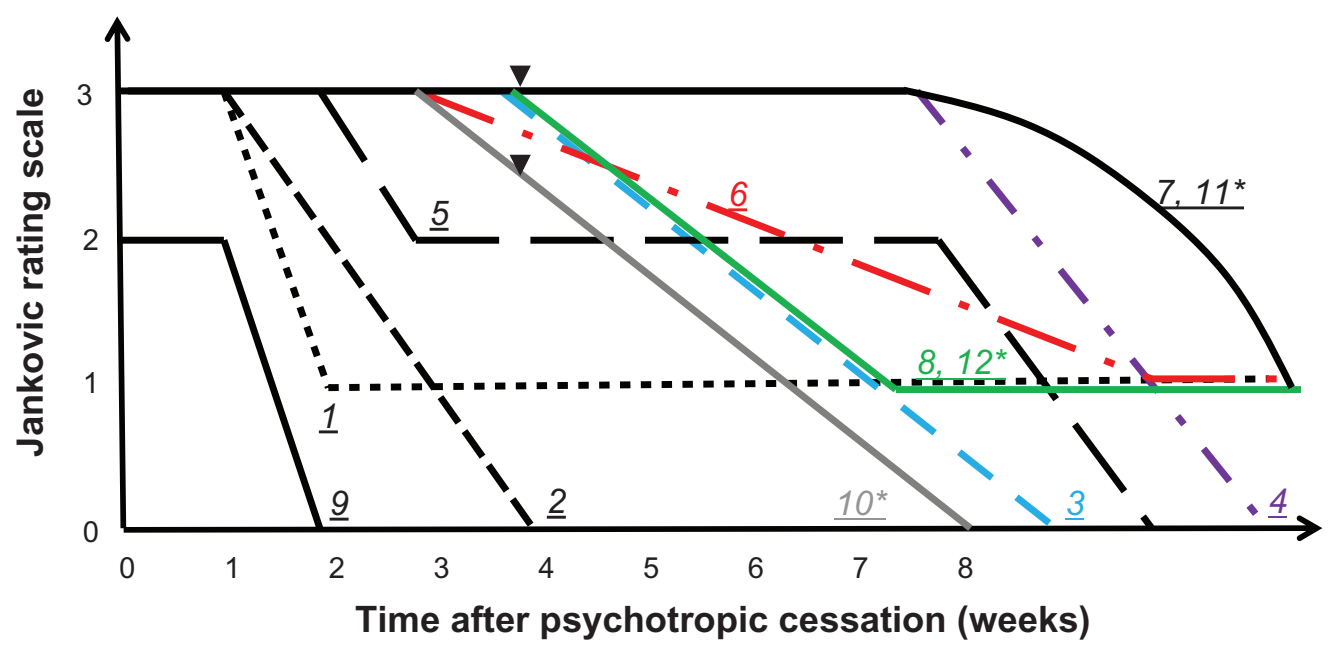

Figure I Clinical course of twelve cases of psychotrophic-induced blepharospasm. After cessation, symptoms started to improve in 3.9 weeks on average (range I-8 weeks). While the effect of the cessation was variable, symptoms improved to more than 2 on the Jankovic scale within 2 months in all cases. Notes: The underlined number in italics indicates the case number.

*Cases 10 and $\mathrm{II}$ received a single botulinum neurotoxin injection 4 weeks after psychotropic cessation ( $\nabla$ ), and Case 12 received an injection 8 weeks before the cessation. 
after cessation. While the effect of psychotropic cessation was variable, symptoms eventually improved to more than 2 on the rating scale. Moreover, psychotropic cessation led to improvement, with complete reversal in eight of twelve patients $(67 \%)$.

Cases 10, 11, and 12 underwent botulinum neurotoxin injection therapy. Cases 10 and 11 received the injection after dose reduction had started, while Case 12 received an injection before dose reduction. After cessation, these three cases started to improve at weeks 3, 8, and 4, respectively. Their symptoms improved to more than 2 on the rating scale. Botulinum neurotoxin injection therapy has been shown to improve the motor symptoms of blepharospasm, ${ }^{3}$ so improvement in these cases was due to both psychotropic cessation and injection therapy. However, the beneficial effect of botulinum neurotoxin is transient. While these three patients only received one injection, they did show improvement after psychotropic cessation and no longer needed injection therapy 1 year after cessation. In the other nine cases, results were similar, with symptoms starting to improve within 2 months of cessation (mean 3.6, range 1-8 weeks). Although our results indicate that psychotropic cessation may have had a greater effect in three cases, we could not determine whether botulinum neurotoxin injection prompted the recovery or whether it was psychotropic cessation that assisted in resolution of the drug-induced blepharospasm.

While psychotropic reduction or cessation would seem to be a logical first step in the management of tardive dystonia and drug-induced blepharospasm, this is not always possible in a clinical setting because of the overriding need to manage current psychotic symptoms and/or reduce the risk of relapse. ${ }^{7}$ We previously showed that there was a high incidence $(68 \%)$ of mood disorders in patients with possible drug-induced blepharospasm when using the Center for Epidemiologic Studies Depression Scale, while there was a $32 \%$ incidence in drug-free blepharospasm versus $16 \%$ in healthy controls. ${ }^{8}$ Thus, there is certainly a risk of depression or mood disorders when blepharospasm is present. Therefore, in patients being treated with minor tranquilizers for mild mental illnesses, such as anxiety or insomnia, psychotropic use should be reduced or discontinued in cases where there is presumed drug-induced blepharospasm.

Although the pathogenesis of drug-induced blepharospasm is not known, abnormalities of the cortical or subcortical neural pathways have been suggested. ${ }^{9}$ With regard to neuroleptics, it has been theorized that the likely mechanism is supersensitivity of striatal dopamine receptors, although this hypothesis has been extensively debated. ${ }^{10}$ Chronic use of these agents may lead to alteration of the normal balance between D1 and D2 receptors, which could cause striatal disinhibition of the thalamocortical pathway and consequent dystonia. ${ }^{11}$ A previous positron emission tomography study suggested that decreased dopamine D2 receptor binding may be one of the predisposing factors leading to dysfunction of the motor circuit, resulting in loss of broad inhibition of unwanted movements during an intended movement in blepharospasm patients. ${ }^{12}$ Unfortunately, these hypotheses do not address what is responsible for causing a proportion of patients treated with neuroleptics to be vulnerable to the development of blepharospasm and other dystonias. ${ }^{11}$

In conclusion, psychotropic medication can effectively control psychiatric symptoms and so has been widely used. However, these drugs can not only cause blepharospasm, but may also exacerbate symptoms. In this study, while the effect of psychotropic reduction or cessation in cases of drug-induced blepharospasm was variable, all cases eventually started to show improvement of symptoms within 2 months. Patients with blepharospasm are occasionally treated with neuroleptics and/or anxiolytics. These agents may be responsible for underlying mental disorders or psychiatric symptoms associated with blepharospasm. Clinicians should consider reducing psychotropic medications, provided the clinical situation permits such a course of action in their patients.

\section{Disclosure}

The authors report no conflicts of interest in this work.

\section{References}

1. Hall TA, McGwin G Jr, Searcey K, Xie A, Hupp SL, Kline LB. Healthrelated quality of life and psychosocial characteristics of patients with benign essential blepharospasm. Arch Ophthalmol. 2006;124(1): 116-119.

2. Hallett M, Evinger C, Jankovic J, Stacy M. Update on blepharospasm: Report from the BEBRF International Workshop. Neurology. 2008; 71(16):1275-1282.

3. Hallett M. Blepharospasm: Recent advances. Neurology. 2002; 59(9):1306-1312

4. Lee Y, Yeh WC, Chong MY, Lin PY, Chang YY. Venlafaxine and tardive blepharospasm: A case report. Prog Neuropsychopharmacol Biol Psychiatry. 2007;31(5):1139-1140.

5. Wakakura M, Tsubouchi T, Inouye J. Etizolam and benzodiazepine induced blepharospasm. J Neurol Neurosurg Psychiatry. 2004; 75(3):506-507.

6. Jankovic J, Orman J. Botulinum A toxin for cranial cervical dystonia: A double-blind, placebo-controlled study. Neurology. 1987; 37(4):616-623.

7. Soares-Weiser K, Rathbone J. Neuroleptic reduction and/or cessation and neuroleptics as specific treatments for tardive dyskinesia. Cochrane Database Syst Rev. 2006;(1):CD000459.

8. Wakakura M. Nascent psychosomatic medicine in ophthalmology. Jpn J Psychosom Med. 2010;50(1):37-42. 
9. Mauriello JA Jr, Carbonaro P, Dhillon S, Leone T, Franklin M. Drug-associated facial dyskinesias. A study of 238 patients. J Neuroophthalmology. 1998;18(2):153-157.

10. Jankovic J. Tardive syndromes and other drug-induced movement disorders. Clin Neuropharmacol. 1995;18(3):197-14.
11. Sachdev P. Tardive blepharospasm. Mov Disord. 1998;13(6): 947-951.

12. Horie C, Suzuki Y, Kiyosawa M, et al. Decreased dopamine D receptor binding in essential blepharospasm. Acta Neurol Scand. 2009; 119(1):49-54

\section{Publish your work in this journal}

Drug, Healthcare and Patient Safety is an international, peer-reviewed open-access journal exploring patient safety issues in the healthcare continuum from diagnostic and screening interventions through to treatment, drug therapy and surgery. The journal is characterized by the rapid reporting of reviews, original research, clinical, epidemiological and post-marketing surveillance studies, risk management, health literacy and educational programs across all areas of healthcare delivery. The manuscript management system is completely online and includes a very quick and fair peer-review system. Visit http://www.dovepress.com/ testimonials.php to read real quotes from published authors. 